[0212-7199 (2007) 24: 11; pp 520-524] ANALES DE MEDICINA INTERNA Copyright (C) 2007 ARAN EDICIONES, S.L.

AN. MED. INTERNA (Madrid) Vol. 24, N. ${ }^{\circ} 11$, pp. 520-524, 2007

\title{
Estudio de las interconsultas solicitadas por el Servicio de Cirugía General al Servicio de Medicina Interna
}

\author{
J. SOLER-BEL, C. SALA, N. RELAÑO, M. J. MOYA, L. VERGÉZ, A. FLOR \\ Servicios de Medicina Interna y Medicina de Familia y Comunitaria.Xarxa Assitencial \\ de Manresa. Barcelona
}

\begin{abstract}
ANALYCES OF THE CONSULTS FROM THE DEPARTMENT OF GENERAL SURGERY TO THE DEPARTMENT OF INTERNAL MEDICINE
\end{abstract}

\section{RESUMEN}

Fundamento y objetivo: Conocer mejor las características de las interconsultas solicitadas por el servicio de Cirugía General a un servicio de Medicina Interna, valorar los resultados y la experiencia desde la visión del internista.

Pacientes y método: Estudio prospectivo de las 129 interconsultas de pacientes ingresados en el servicio de Cirugía General solicitadas durante el año 2005 al servicio de Medicina Interna.

Resultados: Equivalen al 4\% de todos los ingresos del servicio de Cirugía General (el 6,5\% de los pacientes ingresados desde urgencias y el $2 \%$ de los ingresados de forma programada). La media de edad de los pacientes fue de 74 años. Los pacientes fueron visitados una media de 3,3 días. Las causas más frecuentes que motivaron la solicitud fueron la disnea (29\%), la fiebre (21\%), la tos (11\%) y las arritmias $(8 \%)$. Los diagnósticos más frecuentemente realizados fueron la insuficiencia cardiaca $(17 \%)$, la enfermedad pulmonar obstructiva crónica $(15 \%)$, la sobreinfección respiratoria y/o acumulación de secreciones bronquiales (13\%), la neumonía (9\%) y la fibrilación auricular (7\%). El 31\% de los diagnósticos ha correspondido a agudización de enfermedad crónica previamente conocida. Los fallecimientos han sido 19 (15\%), porcentaje mayor al global en el servicio de Cirugía General (2\%). La media de edad de los fallecidos fue de 82 años. La mortalidad fue mayor cuando el ingreso se realizó desde urgencias (17 de 91) que cuando se realizó de forma programada ( 2 de 38 ). La causa más frecuente ha sido el fallo de sutura con peritonitis y sepsis en 5 pacientes.

Conclusiones: La labor del internista no sólo se ha dirigido al tratamiento de las enfermedades crónicas, sino que también ha ayudado en el diagnóstico y tratamiento de procesos agudos relacionados o no con el motivo inicial de ingreso. A destacar la nula especificidad de la tos como síntoma guía en el diagnóstico de pacientes previamente intervenidos. Las causas del fallecimiento se han relacionado casi exclusivamente con el proceso que requirió el ingreso en Cirugía General o las complicaciones de éste (17 de 19).

PALABRAS CLAVE: Interconsulta médica. Medicina Interna. Cirugía General. Interconsulta. Calidad asistencial.

\section{ABSTRACT}

Rational and aim: to better understand the nature of the consults solicited by the Department of General Surgery to the Department of Internal Medicine and to examine the results and the experience from the point of view of an Internal Medicine specialist.

Patients and methods: Prospective analysis of the 129 consults from patients admitted in General Surgery to the Department of Internal Medicine during 2005.

Results: The number of consults was $4 \%$ of all patients admitted in General Surgery $16,5 \%$ of de admitted patients were from the emergency service and $2 \%$ from the planning programme). The median age was 74 years. The patients were visited a mean of 3.3 days. The most frequent reasons for consultation were dyspnea (29\%), fever $(21 \%)$, cough $(11 \%)$ and dysrhythmias (8\%). The most frequent diagnosis were cardiac failure $(17 \%)$, chronic obstructive pulmonary disease (15\%), respiratory infection and/or accumulation of bronchial secretions (13\%), pneumonia (9\%) and atrial fibrillation (7\%). In $31 \%$ of cases the diagnosis was decompensation of previously diagnosed chronic disease. Nineteen patients died (15\%), higher than the global average admitted in General Surgery $(2 \%)$. The average age of these patients was 82 years. The mortality was higher when the admission was from the emergency service (17 of 91) than when it was from the planning programme. The most frequent cause of death was suture failure and peritonitis with secondary septicemia in 5 patients.

Conclusions: The task of the Internal Medicine specialist was not only the treatment of chronic diseases, but also to assist in the diagnosis and treatment of acute diseases related or unrelated to the cause of the admission. To point up the invalid specificity of cough as a guide symptom in the diagnosis of previously operated patients. The causes of death were almost exclusively related to the disease that resulted in the admission to the Department of Surgery or with its complications (17 of 19).

KEY WORDS: Medical interconsultation. Internal Medicine. General Surgery. Consultation. Care quality. 


\section{INTRODUCCIÓN}

La relación entre los diferentes servicios asistenciales hospitalarios se fundamenta principalmente en la interconsulta (IC) médica. Una de las aportaciones del médico internista, que mejora la calidad asistencial, incluye la respuesta a las ICs generadas por otras especialidades.

Estamos asistiendo a un envejecimiento de la población y a un aumento de la complejidad del enfermo. Para los servicios quirúrgicos y sus pacientes es fundamental la resolución correcta de las ICs realizadas a los servicios médicos $(1,2)$. Sin embargo, a pesar de su interés, pocos son los trabajos que la han estudiado y probablemente está siendo infravalorada en la actualidad. Para el servicio de Medicina Interna la actividad derivada de la IC supone un reto y a la vez una importante carga de trabajo.

El objetivo de nuestro trabajo es conocer mejor los problemas médicos consultados a Medicina Interna, de los enfermos ingresados en el servicio de Cirugía General y Digestiva. Se detallan y valoran los resultados del estudio así como la experiencia desde la visión del internista.

\section{PACIENTES Y MÉTODOS}

El estudio se ha llevado a cabo en el Hospital Althaia Xarxa Assistencial de Manresa (Barcelona), que dispone de 420 camas para la hospitalización de enfermos agudos de todas las especialidades médico-quirúrgicas excepto cirugía cardíaca, cirugía torácica, neurocirugía y grandes quemados. Anualmente realiza unas 26.000 altas de pacientes ingresados y es la institución de referencia de una población de 215.000 habitantes. Se han analizado de forma prospectiva las ICs solicitadas por el servicio de Cirugía General y Digestiva (CG) al servicio de Medicina Interna (MI) durante 12 meses (del 1 de noviembre del 2004 al 31 de octubre del 2005). El servicio de CG realizó 3.260 altas (330 de cirugía mayor ambulatoria) durante el año 2005, con unas estancias totales de 15.735 y una estancia media por paciente de 5,4 días. La mortalidad fue del $2 \%$. El peso medio según la casuística del GRD (grupos relacionados con el diagnóstico) del servicio de CG fue de 1.082.

Durante la realización del estudio, la práctica totalidad de las ICs de pacientes ingresados solicitadas por el servicio de CG fueron dirigidas al servicio de MI, excepto el control de la diabetes (endocrinología) y el control del tratamiento con anticoagulantes orales (hematología).

Todas las ICs se resolvieron por el mismo médico adjunto de MI, excepto durante el período vacacional. Prácticamente todas ellas se atendieron en el mismo día si se solicitaban durante los días laborables de las 8 a las 16 horas. Se obtuvo información actualizada de todas las ICs pendientes desde cualquier terminal del hospital. El médico internista consultor, además, realizaba las ICs de los demás servicios del hospital y llevaba un buscapersonas para los requerimientos urgentes. Las ICs urgentes solicitadas en otros horarios y durante los sábados y festivos fueron atendidas, inicialmente, por el médico del Servicio de Urgencias. Posteriormente, si se solicitaba IC a Medicina Interna, era el médico internista consultor quien realizaba el seguimiento.

En muchos casos, la realización de la IC ha requerido una primera visita, solicitud de exploraciones urgentes y/o inicio de tratamiento, y una segunda visita el mismo día para valorar el resultado de las exploraciones y/o la evolución clínica con el tratamiento administrado. Se llevó a cabo el seguimiento del paciente hasta la finalización del proceso que generó la IC o de otros procesos médicos que pudieran haber surgido durante dicho seguimiento. El médico internista consultor, después de la valoración, diagnóstico y tratamiento iniciales, podía solicitar, si lo creía oportuno, IC a otros servicios médicos para el seguimiento posterior del paciente.

Con la intención de facilitar y simplificar la interpretación de los resultados del estudio, se ha considerado: una sola IC, un solo motivo de IC y un solo diagnóstico por paciente. Siempre se ha valorado el proceso más grave. Asimismo, se ha considerado un solo diagnóstico de la patología quirúrgica que generó el ingreso del paciente. Solamente han sido contabilizados los días en que el paciente ha sido visitado por el médico de MI.

\section{RESULTADOS}

Durante los 12 meses de nuestro estudio se contabilizaron 129 ICs del servicio de CG al servicio de MI, que correspondieron al 4\% de todos los ingresos de CG (el 6,5\% de los pacientes ingresados desde urgencias y el $2 \%$ de los pacientes ingresados de forma programada). Supuso el $31 \%$ de todas las ICs realizadas por los diferentes servicios quirúrgicos del hospital a MI. El servicio de CG fue el segundo servicio con más ICs realizadas, después del servicio de Cirugía Ortopédica y Traumatológica. El 93\% (120) de todas las ICs fueron resueltas por el mismo médico adjunto de MI. El 52\% (67) de las IC correspondieron a mujeres y el $48 \%$ (62) a hombres. La media de edad fue de 74 años (30-95). El 54\% (70) de los pacientes incluidos en este grupo fueron intervenidos durante el ingreso. El 70,5\% (91) de los pacientes habían ingresado desde urgencias y solamente el $29,5 \%$ (38) fueron ingresos programados. En 23 pacientes $(18 \%)$ fue requerido inicialmente al médico de guardia para la primera visita en el periodo de tiempo comprendido entre las 16 horas y las 8 horas del día siguiente o en los sábados y festivos. Los pacientes fueron visitados una media de 3,3 días. El 50\% de las ICs fueron resueltas en uno o dos días y el 14\% requirieron 7 o más días de visita. Sólo 3 pacientes fueron trasladados al servicio de Medicina Interna. En 3 pacientes se solicitó por el mismo médico de MI una IC al servicio de Cardiología para el seguimiento del paciente, y en otros cuatro pacientes más se solicitó respectivamente consulta a neumología, nefrología, digestivo y neurología. Tan sólo en 9 pacientes (7\%) se realizó una segunda IC de CG para MI.

La carga de trabajo calculada para el médico de MI fue de un mínimo de 100 minutos diarios de media, repartidos entre las 8 y las 16 horas.

Las causas que motivaron la solicitud de IC están resumidas en la tabla I.

Los diagnósticos más frecuentes realizados en relación al motivo de la IC están resumidos en la tabla II.

El 31\% de los diagnósticos (40) ha correspondido a agudización de enfermedad crónica previamente conocida. De todos ellos, sólo 10 pacientes habían ingresado de forma programada. Los otros 30 pacientes habían ingresado desde urgencias.

El 25\% de las ICs fueron de causa infecciosa. La sobreinfección respiratoria y/o broncoplejia no se ha contabilizado como patología infecciosa. 


\section{TABLA ।}

MOTIVOS DE LAS INTERCONSULTAS

\begin{tabular}{lcc}
\hline Motivos & Número & $\%$ \\
\hline Disnea o insuficiencia respiratoria & 37 & $29 \%$ \\
Fiebre & 27 & $21 \%$ \\
Tos o secreciones audibles & 14 & $11 \%$ \\
Taquiarritmia & 10 & $8 \%$ \\
Hipotensión arterial o shock & 5 & $4 \%$ \\
Control paciente con pluripatologia & 4 & $3 \%$ \\
Desorientación o agitación & 4 & $3 \%$ \\
Vértigo, mareo o síncope & 3 & $2 \%$ \\
Edemas & 3 & $2 \%$ \\
Hipertensión arterial & 2 & \\
Dolor torácico & 2 & \\
Hiponatremia & 2 & \\
Diarrea & 2 & \\
Artritis aguda & 2 & \\
Oliguria & 2 & \\
Hemiparesia & 2 & \\
Otros motivos & 8 & \\
Total de interconsultas & 129 & $100 \%$ \\
\hline
\end{tabular}

TABLA ॥ DIAGNÓSTICOS DE LAS INTERCONSULTAS

\begin{tabular}{lcc}
\hline Diagnósticos & Número & $\%$ \\
\hline Insuficiencia cardiaca & 22 & $17 \%$ \\
Enfermedad pulmonar obstructiva crónica & 19 & $15 \%$ \\
Sobreinfección respiratoria y/o broncoplejia & 17 & $13 \%$ \\
Neumonía & 12 & $9 \%$ \\
Taquiarritmia (fibrilación auricular) & 9 & $7 \%$ \\
Sepsis grave o shock séptico & 7 & $5 \%$ \\
Infección de catéter venoso & 5 & $4 \%$ \\
Absceso abdominal & 4 & $3 \%$ \\
Síndrome confusional agudo & 3 & $2 \%$ \\
Sindrome febril agudo no filiado & 3 & $2 \%$ \\
Gastroenteritis aguda & 2 & \\
Accidente vascular cerebral & 2 & \\
Hipertensión arterial & 2 & \\
Colangitis & 2 & \\
Hipotensión ortostática & 2 & \\
Hiponatremia por SIADH y sueroterapia & 2 & \\
Vértigo posicional & 2 & \\
Tromboembolismo pulmonar & 2 & \\
Insuficiencia renal & 2 & \\
Otros diagnósticos & 10 & \\
Total de interconsultas & 129 & $100 \%$ \\
\hline SADH Secion & &
\end{tabular}

SIADH: Secreción inadecuada de hormona antidiurética.

De las 12 neumonías diagnosticadas, en 8 casos el paciente fue intervenido de patología abdominal con anestesia general de 2 a 7 días antes del diagnóstico. De los 7 pacientes con sepsis grave, 3 fueron diagnosticados de sepsis de origen biliar, 3 de sepsis de origen abdominal por fallo de sutura y 1 paciente de sepsis abdominal no biliar y sin fallo de sutura previa. Los 3 pacientes consultados con derrame pleural derecho, febrícula y tos en el postoperatorio de colecistectomía fueron diagnosticados de absceso subdiafragmático.
En nuestro grupo de pacientes, los diagnósticos quirúrgicos más frecuentes que habían motivado el ingreso en el servicio de Cirugía General fueron: patología benigna de la vía biliar $29(22 \%)$, neoplasia de colon-recto 16 (12\%), hemorragia digestiva $14(11 \%)$, pancreatitis aguda 9 (7\%), oclusión intestinal $9(7 \%)$, hernias o eventración de pared abdominal 8 (6\%), neoplasia gástrica $6(5 \%)$, neoplasia de páncreas o de vía biliar 5 (4\%), dolor abdominal no filiado 4 (3\%), absceso abdominal $3(2 \%)$ e isquemia intestinal $3(2 \%)$.

Se registraron $19(15 \%)$ fallecimientos, con una media de edad de 82 años (52-95). Las causas de la defunción fueron: fallo de sutura con peritonitis y sepsis grave en 5 pacientes previamente intervenidos de neoplasia de sigma estenosante, neoplasia gástrica, oclusión intestinal por hernia incarcerada, oclusión intestinal por bridas y oclusión intestinal por bezoar; colecistis aguda en 3 pacientes de 88, 89 y 91 años con deterioro neurológico y/o general previo; isquemia intestinal en 2 pacientes; oclusión intestinal por neoplasia de colon y por cuerpo extraño yeyunal con deterioro previo y progresivo posterior a intervención quirúrgica en pacientes de 94 y 89 años; sepsis grave por neumococo de aparente origen abdominal en paciente de 52 años; hemorragia digestiva baja; neoplasia de la vía biliar; abdomen agudo con deterioro progresivo en paciente de 95 años; insuficiencia renal progresiva posterior a intervención quirúrgica en paciente con síndrome de demencia, y probable embolia pulmonar con muerte súbita en paciente de 89 años con neoplasia de recto diagnosticada por rectorragias en la convalecencia de fractura de fémur. El último caso se debió a insuficiencia cardiorrespiratoria progresiva en una paciente de 85 años trasladada previamente al servicio de Medicina Interna, una vez resuelta la pancreatitis aguda leve de etiología biliar. Sólo 2 de los 19 pacientes fallecidos fueron ingresados de forma programada para intervención quirúrgica: un paciente con neoplasia gástrica y otro con coledocolitiasis.

\section{DISCUSIÓN}

Son pocos los estudios que abordan la labor asistencial de la IC hospitalaria. Probablemente se debe tanto a la infravaloración de este aspecto de la medicina, como a las dificultades que comporta la realización de un estudio de este tipo. La estandarización del trabajo y la comparación de los resultados obtenidos resulta complicada debido a las características propias y diferenciales de cada hospital en este ámbito de la IC.

Estudios previos sobre la IC de los servicios quirúrgicos (2-5) no diferencian entre el servicio de Cirugía General (CG) y otros servicios quirúrgicos. En nuestro trabajo nos hemos basado exclusivamente en el servicio de CG. Otros aspectos positivos del trabajo que presentamos serían el englobar la práctica totalidad de las ICs realizadas por el servicio de CG a los servicios médicos convencionales, y que prácticamente todas las ICs han sido resueltas por el mismo médico consultor de MI (93\%), lo que aporta una valiosa coherencia interna al estudio. Todo ello nos ha permitido profundizar más en el conocimiento de este grupo de pacientes.

Se han contabilizado 129 interconsultas, que equivalen al $4 \%$ de todos los ingresos de CG. El 50\% de las ICs han sido resueltas en 1 ó 2 días, los pacientes han sido visitados una media de 3,3 días, que equivaldría a unos 5 días de estancia hospitalaria, y la media de edad de los pacientes fue de 74 
años. Todos estos resultados son similares a los anteriormente publicados $(3,4)$.

En nuestro estudio, los motivos más frecuentes de IC, al igual que en estudios previos (2-6), fueron la disnea (29\%) y la fiebre $(21 \%)$. Menos frecuentes, la tos y/o secreciones respiratorias abundantes y audibles $(11 \%)$, las taquiarritmias $(8 \%)$, la hipotensión arterial y/o shock (4\%), la desorientación o agitación (3\%) y el control del paciente con múltiples patologías o con patología basal crónica (3\%).

En este apartado, cabe destacar, la nula especificidad de la tos como síntoma guía en el diagnóstico de pacientes previamente intervenidos. La tos es un síntoma muy frecuente en el postoperatorio de cirugía abdominal y en algunas ocasiones, incluso, puede confundir y orientar equivocadamente hacia una patología banal respiratoria cuando el problema grave se desarrolla a nivel intraabdominal.

Los diagnósticos más frecuentes realizados en relación al motivo de la IC fueron, como es conocido (2-6), la insuficiencia cardiaca (17\%), la enfermedad pulmonar obstructiva crónica $(15 \%)$ y las neumonías $(9 \%)$, pero también un cuadro clínico que hemos definido como sobreinfección respiratoria y/o acumulación de secreciones bronquiales o broncoplejia en pacientes sin EPOC y sin insuficiencia cardiaca (13\%). Otros diagnósticos han sido la fibrilación auricular rápida $(7 \%)$, la sepsis grave o shock séptico $(5 \%)$, la infección por catéter venoso $(4 \%)$ y los abscesos abdominales $(3 \%)$.

Sólo la tercera parte de las ICs $(31 \%)$ fue motivada por enfermedades crónicas conocidas o la descompensación de éstas. Las otras dos terceras partes fueron debidas a procesos agudos, como complicación directa o no de la enfermedad o tratamiento que motivó el ingreso en CG. Algunos de estos procesos agudos son muy graves y requieren un diagnóstico y tratamiento precoz. La labor del internista, por lo tanto, no únicamente fue dirigida al tratamiento de las enfermedades crónicas agudizadas, sino que también ayudó en el diagnóstico y/o tratamiento de procesos agudos relativamente frecuentes como neumonías, y en procesos relacionados directamente con el motivo inicial de ingreso en CG o con el tratamiento de éste, como abscesos intraabdominales en pacientes previamente intervenidos o sepsis grave secundaria a colecistitis aguda, colangitis o a fallo de sutura.

Por todo ello, creemos que la experiencia, implicación y seguridad del médico consultor de MI en este tipo de pacientes es indispensable para permitirle discernir entre la patología infecciosa intra o extraabdominal y ayudar al cirujano, con el diagnóstico precoz, a disminuir la morbimortalidad de los procesos agudos graves.

En este grupo de pacientes, los fallecimientos durante todo el ingreso hospitalario fueron $19(15 \%)$, porcentaje mucho mayor al global en el servicio de CG (2\%). La mortalidad fue mayor cuando el ingreso se realizó desde urgencias (17 de 91) que cuando se realizó de forma programada (2 de 38). La edad media de estos pacientes fue de 82 años, ocho años mayor que la media de todos los pacientes en que se realizó una IC. Las causas del fallecimiento se han relacionado casi exclusivamente con el proceso que requirió el ingreso en $\mathrm{CG}$ o con las complicaciones del tratamiento quirúrgico. El fallo de sutura fue la causa más frecuente de defunción. La descompensación de la enfermedad crónica que el paciente pudiera tener fue generalmente tratada y controlada adecuadamente. Sin duda, la edad extrema de la vida, un nivel basal de salud precario y las enfermedades crónicas severas, principalmente la demen- cia, han influido en el tratamiento menos agresivo o en algunos casos paliativo y en la evolución desfavorable.

En nuestro caso, la relación del internista con el servicio de CG ha sido satisfactoria. Probablemente ello se deba a diversos factores tales como: el largo periodo en la rotación del mismo médico internista como consultor y su motivación, el respeto mutuo por el trabajo del compañero, la rapidez en la respuesta, el seguimiento continuo del paciente y la responsabilidad compartida.

Los conocimientos y habilidades del cirujano y el internista son diferentes y al mismo tiempo complementarios. Conseguir una buena relación entre ambos no es difícil y es el primer paso para mejorar la atención clínica de este grupo de pacientes. Durante nuestro estudio, el cirujano detectó correctamente los problemas médicos y el internista dio una rápida respuesta al requerimiento. Las recomendaciones y tratamientos indicados por el internista, a diferencia de algún estudio previo, fueron seguidas sin ningún problema. El paciente tenía dos médicos que se sentían responsables y que trabajaban en equipo. Siempre que fue posible, el internista definió claramente en el curso clínico los problemas, los diagnósticos y el tratamiento a realizar, y fue actualizándolo día a día. En los pacientes en que se llevó a cabo seguimiento clínico, hubo entre los dos médicos la comunicación oral o enlace oral directo que se comenta en estudios previos $(2,3)$. En la mayoría de los informes de alta, a diferencia también de otros estudios (4), consta, resumido como es costumbre en los servicios quirúrgicos, la evolución, el diagnóstico y el tratamiento relacionado con la IC de MI. Sólo un 7\% de los pacientes requirieron la realización de una segunda IC. Ello evidencia, probablemente, un seguimiento muy correcto del paciente.

Algunas diferencias entre nuestro estudio y los previos se deben, posiblemente, a que en estos últimos se analizan todos los servicios quirúrgicos en general (2-4) o al servicio de Cirugía Ortopédica y Traumatológica en particular $(7,8)$ y en el nuestro exclusivamente el servicio de CG. En nuestro hospital, el control de la diabetes (enfermedad crónica) está a cargo del Servicio de Endocrinología y quizás por ello predominan claramente las ICs de procesos agudos.

Otros estudios han evidenciado una clara mejora de la calidad asistencial prestada con la integración de internistas (7) o geriatras (8) en el servicio de Cirugía Ortopédica y Traumatología, en donde atienden a los pacientes sin necesidad de una IC previa. Conociendo las diferentes características de ambos servicios, creemos que ello no es necesario y probablemente no seria beneficioso en el servicio de CG. En nuestro estudio, como anteriormente se ha resaltado, solamente en el $4 \%$ de los pacientes ingresados en el servicio de CG se solicitó IC a MI. Fue mas frecuente en los enfermos ingresados desde urgencias $(6,5 \%)$ que en los ingresados de forma programada $(2 \%)$. Se trata de un pequeño porcentaje en que la evolución no fue la habitual y/o en que aparecieron algunas dudas o problemas por parte del cirujano. Son pacientes seleccionados por su evolución en los cuales el riesgo de complicaciones graves es muy elevado. Creemos que éstos son los pacientes que deben ser atendidos rápidamente por un internista motivado, responsable y experto. Implicar simultáneamente a múltiples subespecialistas o incluso a diferentes internistas en el manejo de estos pacientes, con la consiguiente disminución de responsabilidad, probablemente generaría cierta confusión, pérdida de tiempo y aumento de la morbimortalidad. 
Sólo $38(29,5 \%)$ del total de las 129 ICs fueron de pacientes ingresados de forma programada, cuando en el total del servicio de $\mathrm{CG}$ predominaban estos ingresos programados $(57 \%)$ sobre los ingresos realizados desde urgencias $(43 \%)$. Únicamente en 10 ICs de las 38 solicitadas de pacientes ingresados de forma programada, el diagnóstico realizado estuvo relacionado con la descompensación de una enfermedad crónica previamente conocida: 4 con EPOC, 3 con insuficiencia cardiaca y 3 con fibrilación auricular. En ningún caso fue problemático el control y tratamiento adecuado de estos procesos. Creemos, por lo tanto, que las interconsultas preoperatorias ordinarias al Servicio

\section{Bibliografía}

1. Girón JA. La interconsulta de servicios quirúrgicos: Una misión para el internista. Rev Clin Esp 2004; 204: 343-4.

2. Díaz JA, Grandes J, Castro B, Cabezas JM, de la Iglesia R, Baeza FJ. Estudio de la interconsulta en un servicio de Medicina Interna. Rev Clin Esp 1999; 199: 66-72.

3. Monte R, Rabuñal R, Rigueiro MT, García MJ, Casariego E, Guerrero J. Papel del internista como consultor de servicios quirúrgicos. Rev Clin Esp 2004; 204: 345-50.

4. Montero J, López J, Hernández C. Estudio de las interconsultas a un servicio de Medicina Interna. Rev Clin Esp 2004; 204: 507-10.

5. García M, Lucena MM, Montero M, Sánchez P. Estudio de la actividad de Anestesia son adecuadas y suficientes, y no estaría justificado, salvo en pacientes muy seleccionados, la IC preventiva al servicio de MI.

El objetivo final de la IC al internista sería la disminución de la morbimortalidad de estos pacientes (1). Supone un reto $\mathrm{y}$, al mismo tiempo, una valiosa aportación del internista, el evitar, en lo posible, el fallecimiento y las complicaciones por enfermedades crónicas descompensadas y por procesos agudos derivados de las mismas enfermedades que han requerido el ingreso en CG. Es un objetivo difícil, pero de importancia capital para la correcta actividad hospitalaria. asistencial de Medicina Interna en relación a las interconsultas. An Med Interna (Madrid) 2003; 20: 510-4.

6. Tudela P, Rego MJ, Tor J, Estrada O, Mòdol JM, Sauquillo JC. Análisis de los avisos internos al equipo de guardia del área médica en un hospital general. Med Clin (Barc) 2000; 114: 730-731.

7. Montero E, Hernández C, López J. Efecto de la adscripción de internistas a un servicio quirúrgico. Med Clin (Barc) 2005; 124: 332-5.

8. González-Montalvo JL, Alarcón T, Sáez P, Bárcena A, Gotor P, Del Río M. La intervención geriátrica puede mejorar el curso clínico de los ancianos frágiles con fractura de cadera. Med Clin (Barc) 2001; 116: $1-5$. 